\title{
Age-dependent ultrastructural changes of coronary artery in spontaneously hypertensive rats
}

\author{
Martina Cebova and Frantisek Kristek \\ Institute of Normal and Pathological Physiology, Centre of Excelence for Cardiovascular Research, Slovak Academy \\ of Sciences, 81371 Bratislava, Slovak Republic
}

\begin{abstract}
The age-dependent differences in basic cardiovascular parameters, geometry and structure of coronary arteries between Wistar and spontaneously hypertensive rats (SHR) were evaluated. SHR of the age 3-, 9-, 17-, and 52-week and age-matched Wistar rats were used. Blood pressure (BP) was measured by the plethysmographic method. Animals were perfused with a glutaraldehyde fixative under pressure of $90 \mathrm{mmHg}$ (3-week-old) and $120 \mathrm{mmHg}$ (9-, 17-, 52-week-old). Coronary arteries were processed for electron microscopy. The proportions and cross sectional areas (CSA) of extracellular matrix in intima and media, endothelial and muscle cells were determined by point counting method. Cardiac hypertrophy and except of 3-week-old rats also BP increase and coronary wall hypertrophy was found in all ontogenic periods in SHR compared to Wistar rats. Arterial wall hypertrophy was evoked by increase of CSA of medial extracellular matrix and smooth muscle cells. In 52-week-old SHR, CSA of muscle cells did not differ from that in 17-week-old SHR but the CSA of intimal and medial extracellular matrix significantly increased. The CSA of endothelial cells and CSA of intimal extracellular matrix were increased only in 52-week-old SHR. The independency between BP and trophicity of individual components of the coronary wall during ontogeny of SHR was documented.
\end{abstract}

Key words: Spontaneously hypertensive rats - Coronary artery - Ultrastructure - Geometry - Ontogeny

Abbreviations: BP, blood pressure; BW, body weight; CSA, cross sectional area; ECM, extracellular matrix; HW, heart weight; SHR, spontaneously hypertensive rats; SP, systolic pressure.

\section{Introduction}

The risk of fatal cardiovascular events is associated with an adverse structural remodeling of the arteries and myocardium, which is frequently caused by hypertension. It is complex with a highly variable phenotype that is influenced by genetic and environmental components. However, the fundamental causes of essential hypertension are still largely unknown. Hypertension leads to adverse remodeling in the arterial wall mainly observed in large elastic arteries (Olivetti et al. 1982). These arteries should no longer be considered

Correspondence to: Frantisek Kristek, Institute of Normal and Pathological Physiology, Slovak Academy of Sciences, Sienkiewiczova 1, 81371 Bratislava, Slovak Republic

E-mail: Frantisek.kristek@savba.sk passive conduits, but rather in terms of their active behavioral response to the mechanical forces to which they are subjected reflecting changing patterns in the structural remodeling of the vessels (Bouvet et al. 2005). Rat models have long been used to study the physiology and pharmacology of cardiovascular function and disease. Data can now be used to better define gene-environment interaction and to facilitate the discovery of new therapeutics. Spontaneously hypertensive rats (SHR) represent the animal models mostly used for human essential hypertension. In adult SHR, elevated pressure has been attributed to abnormalities of both blood vessel wall and sympathetic nervous system, and also a causal connection between vascular hypertrophy, sympathetic nervous system activity and sustained hypertension has been postulated (Folkow 1982). There is a general agreement that there are changes in the geometry of the arterial wall, 
which are associated with established hypertension (Folkow 1982). However, studies have not determined unequivocally if these changes are related to the etiology of hypertension or if they simply represent the response of the arterial wall to elevated arterial blood pressure (BP) or a combination of the two. From several studies in adult SHR with established hypertension, it is difficult to distinguish whether the abnormalities present are causally linked or simply associated (Ashton 2000; Touyz 2005). There is conflicting evidence concerning the sequence of events in the development of hypertension. Two widely held opinions are that structural changes are secondary to a rise in $\mathrm{BP}$, and that the rise of $\mathrm{BP}$ is a consequence of some specific structural changes. Changes that are present before the elevation of the BP are consider primary changes, as compared with secondary changes that are subsequent to the elevation of the BP. However, whether in both essential hypertension and genetic hypertensive rat models, the structural changes precede the increase in BP and remain unknown. There are controversal data about the alterations of the cardiovascular system during the early prehypertensive period of ontogenic development. Whether pathological changes of the cardiovascular system, which are characteristic for adult SHR, are presented in the prehypertensive period of ontogenic development of SHR, or whether these changes are as a consequence of BP elevation are shown only in a later period of ontogeny. The experiments were performed on SHR because hypertension develops gradually over weeks, making it possible to study structural alterations of the arterial wall compared with those of normotensive Wistar rats, if any, at normal BP. Besides, SHR have been regarded as a model for essential hypertension in humans. The purpose of this study was to compare the ontogenic aspect on the remodeling of the arterial wall in SHR. The basic functional parameter of the cardiovascular system and the geometry of the septal branch of the left descending coronary artery in SHR and Wistar rats were determined in four periods of ontogenic development: at the end of lactation, at the beginning of sexual activity, in adult, and at the later period of development of hypertension.

\section{Materials and Methods}

The experiments were carried out in male Wistar and SHR housed in a room with a maintained temperature $\left(22 \pm 2^{\circ} \mathrm{C}\right)$, relative humidity (55 $\pm 10 \%)$, and 12 hour light/dark cycle (lights on at 6:00 a.m.). The animals had free access to standard laboratory chow and water. The experimental protocol for this study was approved by The State Veterinary and Food Administration of the Slovak Republic and followed the recommendation of guidelines for use and care of laboratory animals. Four groups (ten animals in each) of Wistar rats of the age of $3,9,17$, and 52 weeks and the age-matched SHR were taken for the study. In all groups, the systolic pressure (SP) was measured non-invasively in pre-warmed animals by the plethysmographic method on the tail artery each week from 3 to 9 weeks of age, then at the end of the experimental age. Body weight (BW) was recorded at the same time. The structure of the coronary artery was assessed at 3, 9, 17, and 52 weeks of age. At the end of the experiment the animals were sacrificed by an overdose of anesthesia (Rometar and Narkamon, 0.1 and $0.25 \mathrm{ml} / 100 \mathrm{~g}$, respectively) administrated i.p. The chest was opened, a cannula was placed into the left ventricle and the cardiovascular system was perfused at a constant pressure $90 \mathrm{mmHg}$ (3-week-old rats), and 120 $\mathrm{mmHg}$ (the rest of groups) for 10 minutes with a fixative solution (300 mM glutaraldehyde in $100 \mathrm{mM}$ phosphate buffer). The middle part of the septal branch of the left descending coronary artery was excised and immersed in the same fixative overnight, post-fixed with $40 \mathrm{mM} \mathrm{OsO}_{4}$ in $100 \mathrm{mM}$ phosphate buffer, stained en block with $1 \%$ uranylacetate, dehydrated by graded alcohol series and propylene oxide, and embedded in Durcupan ACM. Semi-thin sections were cut perpendicularly to the long axis and stained with a methylene blue. Both, the wall thickness (tunica intima + tunica media) and inner circumference were measured by light microscopy. The wall thickness was measured at $45^{\circ}$ intervals around the circumference of the artery. The inner diameter, cross sectional area (CSA) (tunica intima + tunica media), and wall thickness /inner diameter ratio were evaluated from these data. Sections of approximately $70 \mathrm{~nm}$ thickness were cut on an ultramicrotome (Reichert Nova), stained with alkaline lead citrate and examined in a transmission electron microscope (Tesla BS 500). Volume densities of endothelial cells, smooth muscle cells, extracellular matrix (ECM) between endothelial cells and first layer of smooth muscle cells (ECM1) and ECM among smooth muscle cells (ECM2) of the coronary artery were determined by the Weibel point counting method in an electron microscopy (Weibel et al. 1966). In short, the grid was placed on the respective section randomly and 5000 points were counted. Sections from tree blocks from vessels of control and SHR were processed in the same way. From the volume densities the areas of endothelial cells, smooth muscle cells, and ECM were counted. All results are expressed as the mean \pm S.E.M. Measures were tested for statistical differences with one-way analysis of variance (ANOVA) and followed by Bonferroni test. Value of $p<0.05$ was considered significant.

\section{Results}

\section{Physiological and somatic parameters}

Table 1 summarizes the data about SP, BW, heart weight (HW), and relative heart weight (HW/BW). SP measured 
Table 1. Systolic blood pressure (SBP), body weight (BW), heart weight (HW), relative heart weight (HW/BW) of control Wistar rats (WR) and spontaneously hypertensive rats (SHR)

\begin{tabular}{|c|c|c|c|c|c|}
\hline $\begin{array}{c}\text { Age } \\
\text { (weeeks) }\end{array}$ & Group & $\begin{array}{c}\text { SBP } \\
(\mathrm{mmHg})\end{array}$ & $\begin{array}{l}\text { BW } \\
\text { (g) }\end{array}$ & $\begin{array}{l}\text { HW } \\
(\mathrm{mg})\end{array}$ & HW/BW \\
\hline \multirow{2}{*}{3} & WR & $83 \pm 1.9$ & $60 \pm 0.2$ & $260 \pm 20$ & $4.31 \pm 0.24$ \\
\hline & SHR & $84.2 \pm 1.4$ & $32 \pm 1.4^{* *}$ & $250 \pm 10$ & $7.71 \pm 0.15^{\star *}$ \\
\hline \multirow{2}{*}{9} & WR & $107 \pm 1.0$ & $230 \pm 7.6$ & $1.06 \pm 0.05$ & $4.70 \pm 0.25$ \\
\hline & SHR & $154 \pm 1.4^{\star *}$ & $190 \pm 6.8^{\star *}$ & $1.08 \pm 0.02$ & $5.75 \pm 0.12^{\star \star *}$ \\
\hline \multirow{2}{*}{17} & WR & $114 \pm 1.4$ & $423 \pm 3.8$ & $1.35 \pm 0.03$ & $3.13 \pm 0.03$ \\
\hline & SHR & $214 \pm 7.0^{\star *}$ & $324 \pm 5.7^{\star *}$ & $1.59 \pm 0.04^{\star *}$ & $4.91 \pm 0.18^{\star \star}$ \\
\hline \multirow{2}{*}{52} & WR & $115 \pm 3.0$ & $475 \pm 19.0$ & $1.48 \pm 0.09$ & $3.14 \pm 0.09$ \\
\hline & SHR & $190 \pm 2.4^{\star *}$ & $393 \pm 10.0^{* *}$ & $1.91 \pm 0.09^{\star *}$ & $4.86 \pm 0.17^{\star \star}$ \\
\hline
\end{tabular}

Data are means \pm S.E.M.; ${ }^{* *} p<0.01 v s$. corresponding control rats.

from 3 to 9 weeks of age (Fig. 1) was increased in SHR group from the age of 6 weeks $(p<0.01)$. SP of 3 -week-old SHR did not differ from the agematched control Wistar rats. In the 9-, 17-, and 52-week-old animals, SP, measured the day before the end of the experiment, was increased in SHR group compared with the age-matched Wistar rats $(p<0.01)$. In 52-week-old SHR continual increasing of SP, which was observed from the 6th week of age, was stopped, but it was still significantly higher than in age-matched control Wistar rats. BW in both strains gradually increased with the age. Wistar rats are normally heavier than SHRs of the same age, in the present series of the experiment.

$\mathrm{HW}$ of SHRs, after perfusion with the glutaraldehyde fixative, at the age of 3 and 9 weeks did not differ from control Wistar rats in spite of the lower BW. The differ- ences in this respect were found at the age of 17 and 52 weeks in SHR. Owing to unequal increase of the BW and $\mathrm{HW}, \mathrm{HW} / \mathrm{BW}$ was higher in all SHR groups over the control rats.

\section{Morphological parameters}

The individual geometric parameters of the coronary artery (septal branch of the left descending coronary artery) are given in Table 2 .

The inner diameter of the coronary artery in 3- and 9week-old SHR did not differ from inner diameter in control Wistar rats of the same age. Significantly increasing of inner diameter was observed only in 17- and 52-week-old SHR in comparison to the age-matched Wistar rats.

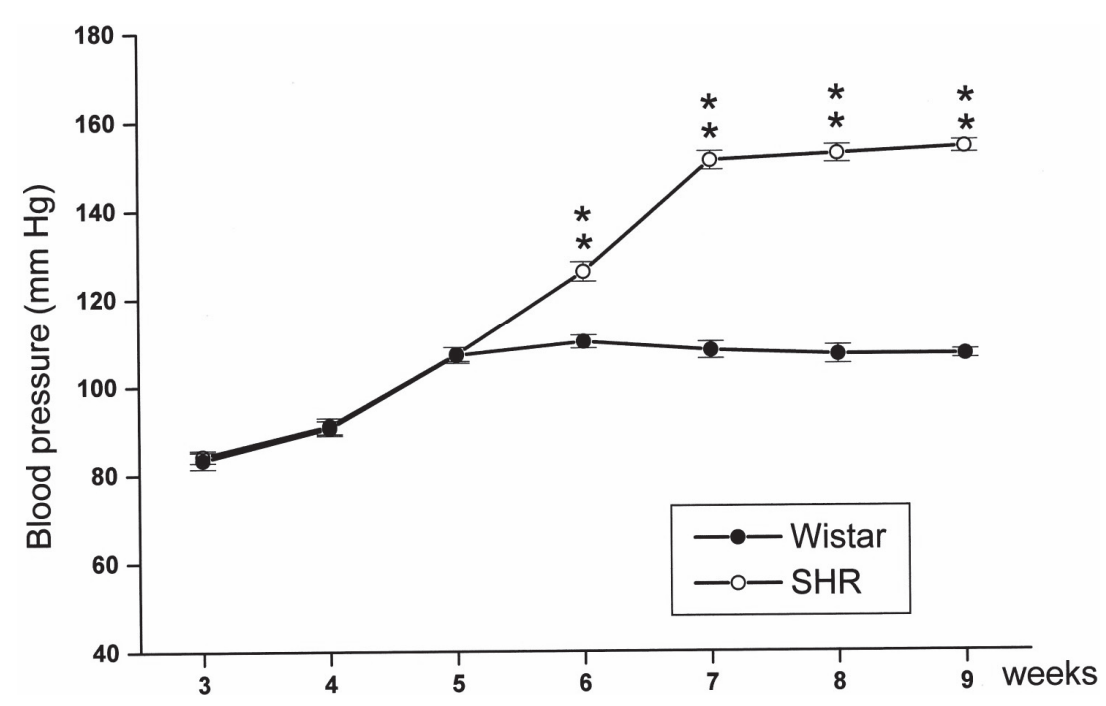

Figure 1. Systolic blood pressure of spontaneously hypertensive rats (SHR) and control Wistar rats (Wistar). The values represent the data at the end of each week from 3 to 9 weeks of age in control Wistar rats and SHR. Data are means \pm S.E.M., ${ }^{* *} p<0.01 v s$. corresponding control rats. 
Table 2. Wall thickness (WT), inner diameter (ID), cross sectional area (CSA), and wall thickness/inner diameter ratio (WT/ID) of septal branch of the left descending coronary artery of control Wistar rats (WR) and spontaneously hypertensive rats (SHR)

\begin{tabular}{|c|c|c|c|c|c|}
\hline $\begin{array}{c}\text { Age } \\
\text { (weeks) }\end{array}$ & Group & $\begin{array}{l}\text { WT } \\
(\mu \mathrm{m})\end{array}$ & $\begin{array}{l}\text { ID } \\
(\mu \mathrm{m})\end{array}$ & $\begin{array}{c}\text { CSA } \\
\left(\mu \mathrm{m}^{2}\right)\end{array}$ & WT/ID $\left(10^{-2}\right)$ \\
\hline \multirow{2}{*}{3} & WR & $9.6 \pm 0.25$ & $182 \pm 6$ & $5790 \pm 240$ & $5.34 \pm 0.20$ \\
\hline & SHR & $10.7 \pm 0.65$ & $161 \pm 9$ & $5760 \pm 590$ & $6.69 \pm 0.48^{\star *}$ \\
\hline \multirow{2}{*}{9} & WR & $10.6 \pm 0.88$ & $220 \pm 11$ & $7660 \pm 620$ & $4.86 \pm 0.64$ \\
\hline & SHR & $13.8 \pm 0.59^{\star *}$ & $212 \pm 5$ & $9800 \pm 390^{* *}$ & $6.60 \pm 0.40^{*}$ \\
\hline \multirow{2}{*}{17} & WR & $9.3 \pm 0.67$ & $250 \pm 12$ & $7580 \pm 840$ & $3.7 \pm 0.23$ \\
\hline & SHR & $20.1 \pm 1.25^{\star *}$ & $321 \pm 26^{\star *}$ & $21630 \pm 2410^{* *}$ & $6.32 \pm 0.71^{\star *}$ \\
\hline \multirow{2}{*}{52} & WR & $10.9 \pm 0.47$ & $286 \pm 12$ & $10260 \pm 680$ & $3.92 \pm 0.22$ \\
\hline & SHR & $20.3 \pm 1.21^{\star \star}$ & $429 \pm 14^{\star *}$ & $28720 \pm 1980^{\star *}$ & $4.77 \pm 0.27^{\star *}$ \\
\hline
\end{tabular}

Data are means \pm S.E.M.; ${ }^{*} p<0.05,{ }^{* *} p<0.01$ vs. corresponding control rats.

The wall thickness of the coronary artery was the same during all ontogenic development in control Wistar rats. In SHR, wall thickness progressively increased with the age. CSA progressively increased with the age in both, SHR and control Wistar rats. The values of both wall thickness and CSA in 3-week-old SHR did not differ from the age-matched control Wistar rats. The differences in this respect were observed from the age of 9 weeks.

The wall thickness/inner diameter ratio of SHR group was significantly increased during the whole ontogenic development in comparison to the age-matched controls.

\section{Cellular vs. extracellular component}

We sought to determine which component of the arterial wall is responsible for increasing the total mass of the coronary artery. The point-counting method gave a comparison between cellular and extracellular components of the arterial wall of SHR and Wistar rats. The results summarized in Table 3 indicate changes in the proportion and areas of individual components of the arterial wall during ontogenic development.

The proportion and area of both, endothelial cells and ECM1 at 3 weeks of the age were the same in SHR as in Wistar rats. The proportion of endothelial cells and ECM1 from 9 weeks was lower in SHR group than in Wistar rats. However, the area of both parameters at 9- and 17-week-old SHR was the same and at 52 weeks it had significantly increased.

The same tendency of 3-week-old animals was found in both proportion and area of smooth muscle cells. At 9- and 17-week-old SHR proportion and area of smooth muscle cells had increased in comparison to the age matched Wistar rats. At 52-weeks SHR showed a decrease of smooth muscle cells proportion than in control, however, the area of smooth muscle cells had still significantly increased considering the total area of the arterial wall.

The proportion of ECM2 was the same between strains but at 52-weeks SHR was twice higher than in the control.

Table 3. Proportion (\%) and cross sectional areas $\left(\mu \mathrm{m}^{2}\right.$ ) of endothelial cells (EC), smooth muscle cells (SMC), extracellular matrix in tunica intima (ECM1), and extracellular matrix in tunica media (ECM2) in septal branch of the left descending coronary artery of control Wistar rats (WR) and spontaneously hypertensive rats (SHR)

\begin{tabular}{|c|c|c|c|c|c|c|c|c|c|}
\hline \multirow{2}{*}{$\begin{array}{c}\text { Age } \\
\text { (weeks) }\end{array}$} & \multirow{2}{*}{ Group } & \multicolumn{2}{|c|}{ EC } & \multicolumn{2}{|c|}{ SMC } & \multicolumn{2}{|c|}{ ECM1 } & \multicolumn{2}{|c|}{ ECM2 } \\
\hline & & $(\%)$ & $\left(\mu \mathrm{m}^{2}\right)$ & $(\%)$ & $\left(\mu \mathrm{m}^{2}\right)$ & $(\%)$ & $\left(\mu \mathrm{m}^{2}\right)$ & $(\%)$ & $\left(\mu \mathrm{m}^{2}\right)$ \\
\hline \multirow{2}{*}{3} & WR & $12.9 \pm 0.7$ & $748 \pm 32$ & $66.9 \pm 1$ & $3873 \pm 164$ & $10 \pm 0.6$ & $578 \pm 24$ & $10.5 \pm 0.5$ & $609 \pm 26$ \\
\hline & SHR & $13.1 \pm 0.9$ & $770 \pm 52$ & $66.3 \pm 1$ & $3899 \pm 266$ & $10.7 \pm 0.5$ & $528 \pm 43$ & $10.1 \pm 0.8$ & $594 \pm 40$ \\
\hline \multirow{2}{*}{9} & WR & $12.1 \pm 0.4$ & $890 \pm 55$ & $68.4 \pm 0.7$ & $5046 \pm 313$ & $8.7 \pm 0.4$ & $638 \pm 40$ & $10.8 \pm 0.4$ & $797 \pm 49$ \\
\hline & SHR & $8.4 \pm 0.5^{\star *}$ & $825 \pm 32$ & $73.3 \pm 0.5^{\star *}$ & $7220 \pm 282^{\star *}$ & $6.9 \pm 0.3^{* *}$ & $675 \pm 26$ & $11.4 \pm 1.4$ & $1125 \pm 44^{* *}$ \\
\hline \multirow{2}{*}{17} & WR & $8.5 \pm 0.7$ & $664 \pm 67$ & $74 \pm 1.1$ & $5772 \pm 862$ & $6.2 \pm 0.4$ & $484 \pm 78$ & $10.9 \pm 1.3$ & $851 \pm 333$ \\
\hline & SHR & $4.6 \pm 0.7^{\star *}$ & $987 \pm 155$ & $81.7 \pm 1.8^{\star *}$ & $17730 \pm 379^{* *}$ & $2.7 \pm 0.5^{\star *}$ & $586 \pm 103$ & $11 \pm 1.3$ & $2383 \pm 277^{\star *}$ \\
\hline \multirow{2}{*}{52} & WR & $8.5 \pm 0.4$ & $872 \pm 55$ & $72.8 \pm 0.7$ & $7468 \pm 539$ & $6.2 \pm 0.3$ & $635 \pm 45$ & $12.5 \pm 0.7$ & $1281 \pm 86$ \\
\hline & SHR & $4.6 \pm 0.3^{\star *}$ & $1315 \pm 156^{*}$ & $64.9 \pm 1.1^{\star *}$ & $18610 \pm 1249^{\star *}$ & $5.4 \pm 0.3^{*}$ & $1550 \pm 147^{\star *}$ & $25 \pm 1^{\star *}$ & $7170 \pm 405^{\star *}$ \\
\hline
\end{tabular}

Data are means \pm S.E.M.; ${ }^{*} p<0.05,{ }^{* *} p<0.01$ s. corresponding control rats. 
However, the area of ECM2 had significantly increased from 9 weeks in SHR in comparison to the control.

\section{Discussion}

This study was investigated at different ages in the areas of basic physiological parameters, geometry, and the ultrastructure of the coronary artery in SHR compared to those of Wistar normotensive rats. The findings in the present study show that in SHR, in which hypertension develops gradually over weeks, alteration in the coronary artery wall properties precede the development of hypertension.

In our experiments, we found that BP in 3-week-old SHR did not differ from the age matched control Wistar rats. These data are in a good agreement with the original description of Okamoto and Aoki (1963), where elevation of BP in SHR was detected from only 5 weeks of age. Nevertheless, there are some concerns whether the prehypertensive period exists in the development of hypertension in SHR, mainly because there are authors showing that BP of SHR was already significantly higher than the age-matched Wistar-Kyoto rats from birth (Gray 1984). However, the study of Lais et al. (1977) in neonatal and young SHR showed that systolic and diastolic BP were similar between SHR and the controls. We have found that the BP of young SHR became higher than age-matched control rats from 6 weeks of age. Similar results in the prehypertensive period have also been reported earlier from other laboratories (Olivetti et al. 1982; Lee 1985; Rizzoni et al. 2000).

There are possibilities for differences, which have been reported in the literature about BP during the prehypertensive period of SHR and control Wistar rats. These differences in the onset of hypertension might be intrastrains differences in SHR used by the various investigators (Dmitrieva et al. 2009) and by different methods of BP measurement. In studies where the BP of neonatal SHR was reported to be higher than age-matched control rats, BP was measured through a cannula placed into the carotid artery (Gray 1984). This is in contrast with the indirect plethysmography methods (Lais et al. 1977; Olivetti et al. 1982; Lee, 1985; and this study) or direct measurement through the femoral artery (Lais et al. 1977) where no difference in BP was found between young SHR and control rats. The differential outcome of the two measurements might be related to the fact that the tail-cuff method records systolic BP in the rat tail, whereas in the direct measure both systolic and mean arterial pressure were recorded at the level of the aortic arch. Alternatively, the differences in two variables might also be influenced by having made these determinations in rats with and without anesthesia.

From the age of 6 weeks SP was remarkably higher in SHR group than in control Wistar rats. Continual increasing of
SP was stopped after 17-weeks in SHR, and SP was dropped in 52-weeks SHR, but it was still significantly higher in comparison to the age-matched normotensive controls. It is in good agreement with results in the literature (Marque et al. 1999). Authors found that the elevated values of SP in SHR have a spontaneous tendency to decline after 36 weeks of age. It might be due to the reduction of cardiac output, which is connected with the age and /or gradual heart failure.

The BW in experimental groups was lower during the entire ontogenic development. Similar results were presented by Cunha et al. (1997) at the age of 5 and 12 weeks, Rizzoni et al. (2000) in 8 and 12 weeks, Van Gorp et al. (2000) in 3 and 6 months, and Bezie et al. (1998) in 52-week-old. SHR usually have a lower mean BW than Wistar-Kyoto rats (Cabassi et al. 1996). Similar lower BW was observed in 4week-old rats from dams, which were administrated with L-NAME (Gerova et al. 2002). Long-term administration of L-NAME evokes hypertension in offspring and adults (Kristek and Gerova 2004).

On the other hand, other authors did not find differences between BW of control Wistar rats and age-matched SHR. Mulvany and Halpern (1977) in 21 weeks, and Marque et al. (1999) in 3, 9, and 15 months. Expressive differences in BW of SHR support that individual lines are different from the original strain of SHR described by Okamoto and Aoki (1963). SHR have different uterine environment from control Wistar-Kyoto rats. Amniotic fluid volume was found to be lower at days 15-18 of gestation in the SHR compared with Wistar-Kyoto rats. Amniotic fluid sodium concentration was comparable between strains, but potassium was lower in the amniotic fluid of SHR dams. Erkadius et al. (1995) associated these differences with reduced fetal growth during the last 2-3 days of gestation. The association between birth weight and the risk of developing cardiovascular disease in adulthood has been reported in populations from several countries. Indeed, low birth weight has been correlated with an increased risk of coronary artery disease and hypertension.

HW/BW is the determinant of cardiac hypertrophy. The morphogenesis of the heart and vessels occurs mainly during the prenatal period, but it continues partially even after birth (Zicha and Kunes 1999). In the period shortly after birth, the heart grows more rapidly than the body so that the $\mathrm{HW} / \mathrm{BW}$ ratio attains its maximum at 4-5 days of postnatal life and than declines (Clubb and Bishop 1984). Despite the diminished cellular division, the number of cardiomyocytes in the heart doubles during the first 3-4 weeks of life (Kunes et al. 1987). Our results are in good agreement with this observation. In both groups of rats we found that a decrease of $\mathrm{HW} / \mathrm{BW}$ depended on the age. In SHR group the HW/BW ratio was significantly higher in all periods of ontogeny. In spite of unchanged SP, cardiac hypertrophy was still found in 3-week-old SHR. Hypertrophy of myocardium in 9-, 17-, 
and 52-week-old SHR is probably the result of an increased workload of the heart and rise of peripheral resistance and increase of BP. Our results suggested that $\mathrm{SP}$ is not necessarily linked together with cardiac hypertrophy.

The results of these experiments have documented a number of differences in the geometry of the coronary artery from Wistar rats and SHR.

Our study shows that at the prehypertensive period of hypertension development where BP in SHR did not differ in comparison to the age-matched Wistar rats, the structural alterations of blood vessels were already found.

The increased inner diameter of the coronary artery in the later period of ontogeny ( 17 and 52 weeks) was accompanied with an increase of BP and arterial wall mass. Keeping mind Laplace's law, the increase of the inner diameter might be adaptation mechanism for the elevation of BP.

The wall thickness of the coronary artery was the same in control Wistar rats during the entire ontogenic development. This measurement showed that the wall thickness from the age of 3 weeks did not differ in spite of the fact that the BW increased nearly 8 times. Our results are in agreement with González et al. (2002) on basilar arteries at the age of 4 and 24 weeks.

Both the wall thickness and CSA of the coronary artery in SHR in the prehypertensive period did not differ from the age-matched control Wistar rats. In this case, term eutrophic remodeling is applicable, when the wall thickness/inner diameter ratio was increased and no changes in the net amount of the arterial wall mass were found. Our results are agreed with the observation of Rizzoni et al. (2000) on mesenteric artery, Bund et al. (1991) on femoral artery in 5-week-old rats. Lee (1985) did not find any changes on mesenteric artery, but in the elastic artery he showed an increase of the arterial wall mass in 3-week-old SHR in comparison to the age-matched Wistar-Kyoto rats.

Changes observed in the coronary artery in the established phases of ontogenic development (9-, 17-, and 52week-old) were mostly due to an increase in the wall thickness and CSA in the SHR as compared to the age-matched Wistar rats. It is probable that changes in the coronary artery of SHR were due to a long duration of hypertension. A gain in wall material, together with an increase of the wall thickness to inner diameter ratio, indicated hypertrophic remodeling of the coronary artery in these stages of hypertension. It is generally accepted that an increase of $\mathrm{BP}$ is accompanied by an increase of the arterial wall mass. The mechanisms, responsible for increasing wall mass in SHR, are yet unknown. The sympathetic nervous system, renin-angiotensin system (RAS), nitric oxide, apoptosis, and cellular $v s$. intercellular components of the arterial wall may be considered.

Hypertrophy of the coronary artery wall during hypertension could be a result of enhanced activity of the sympathetic nervous system, which is known to be present before the onset of BP elevation. This overall increase in activation and sensitivity to the sympathetic nervous system could induce hypertrophy of the smooth muscle cells by its growth promoting effect (Bevan 1984). Due to of the observations that the sympathetic nervous system in young SHR is increased and that the sympathetic nervous system exerts a trophic effect on the development of blood vessels, it is quite possible that the structural alterations of the blood vessels are secondary to the hyperactive sympathetic nervous system. Hyperinnervation of blood vessels has been reported in the muscular mesenteric arteries of young and older SHR (Lee 1985).

A possible role for the RAS cannot be excluded. Saavedra et al. (1992) described an increase in angiotensin-converting enzyme in aorta of the prehypertensive SHR compared with Wistar-Kyoto rats. Activity of the renal RAS in the SHR is greatly increased in the first 2 critical weeks of lactation. However, SHR pups have significantly higher concentrations of renal renin, more that Wistar-Kyoto pups from birth to the beginning of the third postnatal week, as well as increased expression of angiotensinogen mRNA, and renal angiotensin II type 1 receptor density (Correa et al. 1995). The consequence of this up-regulation in renal RAS activity in SHR pups may be gross changes in renal haemodynamics. Harrap et al. (1990) have revealed that the elevated renal renin concentration of the SHR is linked to increased renal vascular resistance and thus to a reduced renal blood flow and glomerular filtration rate. These renal abnormalities have been genetically linked to the development of high $\mathrm{BP}$ in the SHR and brief angiotensin- 1 converting enzyme inhibition, which permanently lowers renal vascular resistance (Harrap et al. 1990).

Angiotensin II has a growth-inducing activity on the vascular smooth cells in cell cultured and on the large and small arteries even at suppressor doses (Boonen et al. 1993). Inhibition of RAS is associated with a reduction of wall thickness. There is increasing evidence that the vascular hypertrophic effect of the sympathetic system and of the RAS may be closely related (Stassen et al. 1997).

Our results are comparable with the results of Cunha et al. (1997) on carotid artery of 12-week-old rats, Kristek et al. (1996) on carotid and coronary arteries of 16-week-old rats, Bund et al. (1991) on femoral artery of 12- and 24-week-old rats, and Clozel et al. (1989) on carotid artery of 14-33week-old rats. Lee (1985) found that medial wall thickness was mainly due to hyperplasia of smooth muscle cells, but later authors corrected this argument (Dickhout and Lee 2000). Previous results were explained by the methods that were used. This is in contrast with our findings. We did not observe any mitosis of smooth muscle cells and therefore, we assume that arterial wall thickening was mainly due to hypertrophy. Owens and Schwartz (1982) also found that 
the increase of the wall mass was due to hypertrophy of the smooth muscle cells.

The question was opened as to which part of the arterial wall mass was really increased: endothelial cells, smooth muscle cells, and/or ECM. Altered vascular smooth muscle cells growth/apoptosis, contraction/relaxation, migration and differentiation, impaired production and degradation of ECM and stimulation of inflammatory responses resulted in vascular remodeling.

Cellular mechanisms and intracellular signaling events implicated in arterial remodeling in hypertension are complex. At the electron microscope level, in the intima (endothelial cells and ECM) and in the media (smooth muscle cells and ECM) neither differed in volume densities or areas of SHR coronary artery at the prehypertensive phase. It corresponded to the re-organization of cellular and noncellular material of existing vascular wall called eutrophic remodeling. Rummery et al. (2002) showed that endothelial cell area, length, width and perimeter of the tail artery were not significantly different between SHR and Wistar-Kyoto rats at 3 weeks of age. Study of Olivetti et al. (1982) revealed similar findings to our results in the volume composition of the smooth muscle cells of the aorta at 21 days after birth. Lee (1985) found similar CSAs of endothelial and smooth muscle cells in the superior mesenteric arteries, but not in the large mesenteric arteries, at the prehypertensive stage between SHR and Wistar-Kyoto rats.

In the intima at a later period of ontogenic development in SHR, a decrease was found in volume densities of endothelial cells and ECM, but there were no changes in the areas of both parameters. At the developing phase, an increase of the arterial wall mass was due to hypertrophy of the smooth muscle cells. A study by Cuspidi et al. (2003) has revealed a similar finding, where in which aging and arterial hypertension are both associated with an increased prevalence of the carotid structural abnormalities, such as intima - media thickening or plaques, which are powerful independent predictors of the cardiovascular events. Michel et al. (1994) found an increase of the intimal space in 24- to 30-month-old normotensive rats. In many experimental models, hypertension thickening of tunica intima is a consequence of an accumulation of proteins and adjunctive tissues in subendothelial cells and could be accompanied by a change of permeability. Permeability of intima is changed depending on component substances such as histamin, serotonin, bradykinin, and angiotensin II (Chobanian 1983).

The increase of the arterial wall mass in SHR during ontogenic development was induced preferentially by an increase of the tunica media. Analysis of the tunica media showed that volume densities and areas of smooth muscle cells were increased in SHR to the age of 17 weeks. After 17 weeks, in spite of the increase of the arterial wall mass in SHR, the area of the smooth muscle cell did not differ in comparison to younger SHR, volume densities actually decreased. The volume densities of ECM in tunica media to the age of 17 weeks did not differ in comparison to the age matched control Wistar rats. After 17 weeks of age it was increased approximately 2.5 -fold as in controls. Areas of ECM in tunica media consistently increased. However, the most expressive increase was after 17 weeks of age. This means that the increase of the arterial wall mass after 17 weeks of age was due to the increased area of ECM. Unfortunately, there are no literary data available for comparison with our results, yet, they are indirectly supported by Schwartz et al. (1990), who showed increase of ECM in the hypertrophy of large arteries in human and animal hypertension. On the other hand, Bezie et al. (1998) did not find changes in the area of ECM in 1-year-old aorta between SHR and control rats. We assume that differences between results can probably be explained by the differences in the types of vascular bed and very likely that the coronary artery was filled during diastole.

In conclusion, the prehypertensive period in SHR was accompanied by cardiac hypertrophy and eutrophic remodeling of arterial wall, despite unchanged BP. In the later period of ontogenic development in SHR we found hypertrophy of the coronary artery, which was evoked by an increasing of the areas of the smooth muscle cells and ECM to the age of 17 weeks. After this time, there was solely an increase in the area of the ECM.

Acknowledgements. The study was supported by VEGA grant 2/0019/10, Slovak Republic.

\section{References}

Ashton N. (2000): Perinatal development and adult blood pressure. Braz. J. Med. Biol. Res. 33, 731-740 http://dx.doi.org/10.1590/S0100-879X2000000700002

Bevan R. D. (1984): Trophic effects of peripheral adrenergic nerves on vascular structure. Hypertension 6, III19-26

Bezie Y., Lacolley P., Laurent S., Gabella G. (1998): Connection of smooth muscle cellls to elastic lamellae in aorta of spontaneously hypertensive rats. Hypertension 32, 166-169

Boonen H. C., Daemen M. J., Eerdmans P. H., Fazzi G. E., van Kleef E. M., Schiffers P. M., De Mey J. G. (1993): Mesenteric small artery changes after vasoconstrictor infusion in young rats. J. Cardiovasc. Pharmacol. 22, 388-395 http://dx.doi.org/10.1097/00005344-199309000-00007

Bouvet C., Gilbert L. A., Girardot D., deBlois D., Moreau P. (2005): Different involvement of extracellular matrix components in small and large arteries during chronic NO synthase inhibition. Hypertension 45, 432-437 http://dx.doi.org/10.1161/01.HYP.0000154680.44184.01

Bund S. J., West K. P., Heagerty A. .M. (1991): Effects of protection from pressure on resistance artery morphology and reactivity in spontaneously hypertensive and Wistar-Kyoto rats. Circ. Res. 68, 1230-1240 
Cabassi A., Bergamaschi E., Mutti A., Franchini I., Borghetti A. (1996): Age-related changes in interstitial norepinephrine. A microdialysis study in spontaneously hypertensive rats. Am. J. Hypertens. 9, 878-883

http://dx.doi.org/10.1016/S0895-7061(96)00094-5

Chobanian A. V. (1983): The influence of hypertension and other hemodynamic factors in atherogenesis. Prog. Cardiovasc. Dis. 26, 177-196 http://dx.doi.org/10.1016/0033-0620(83)90005-1

Clozel J. P., Kuhn H., Hefti F. (1989): Decreases of vascular hypertrophy in four different types of arteries in spontaneously hypertensive rats. Am. J. Med. 87 (suppl 6B), 92S-95

Clubb F. J., Bishop S. P. (1984): Formation of binucleated myocardial cells in the neonatal rat. An index for growth hypertrophy. Lab. Invest. 50, 571-577

Correa F. M., Viswanathan M., Ciuffo G. M., Tsutsumi K., Saavedra J. M. (1995): Kidney angiotensin II receptors and converting enzyme in neonatal and adult Wistar-Kyoto and spontaneously hypertensive rats. Peptides 16, 19-24 http://dx.doi.org/10.1016/0196-9781(94)00150-5

Cunha R. S., Dabire H., Bezie I., Weiss A. M., Chaouche-Teyara K., Laurent S., Safar M. E., Lacolley P. (1997): Mechanical stress of the carotid artery at the early phase of spontaneous hypertension in rats. Hypertension 29, 992-998

Cuspidi C., Michev I., Macca G., Meani S., Salerno M., Valerio C., Lonati L., Leonetti G., Magrini F., Zanchetti A. (2003): Impact of carotid intima-media thickening on risk stratification in elderly hypertensives. Blood Press 12, 25-31

Dickhout J. G., Lee R. M. (2000): Increased medial smooth muscle cell length is responsible for vascular hypertrophy in young hypertensive rats. Am. J. Physiol. Heart Circ. Physiol. 279, H2085-2094

Dmitrieva R. I., Hinojos C. A., Grove M. L., Bell R. J., Boerwinkle E., Fornage M., Doris P. A. (2009): Genome-wide identification of allelic expression in hypertensive rats. Circ. Cardiovasc. Genet. 2, 106-115 http://dx.doi.org/10.1161/CIRCGENETICS.108.809509

Erkadius E., Morgan T. O., DiNicolantonio R. (1995): Amniotic fluid composition and fetal and placental growth rates in genetically hypertensive and normotensive rats. Reprod. Fertil. Dev. 7, 1563-1567 http://dx.doi.org/10.1071/RD9951563

Folkow B. (1982): Physiological aspects of primary hypertension. Physiol. Rev. 62, 347-504

Gerova M., Bernatova I., Torok J., Jurani M. (2002): Cardiovascular system in offsprings of hypertensive rats with defective nitric oxide production. Physiol. Res. 51, 465-474

Gonzalez J. M., Somoza B., Gonzalez M. C., Guerra P., Arribas S. M. (2002): Progression of hemodynamic alterations and remodeling of cerebrál arteries in prehypertensive and adult spontaneously hypertensive rats. J. Hypertens. 20 (Suppl. 4), S187

Gray S. D. (1984): Spontaneous hypertension in the neonatal rat. Clin. Exp. Hypertens. 6, 755-781 http://dx.doi.org/10.3109/10641968409044037

Harrap S. B., van der Merwe W. M., Griffin S. A., Macpherson F., Lever A. F. (1990): Brief angiotensin converting enzyme inhibitor treatment in young spontaneously hypertensive rats reduces blood pressure long-term. Hypertension 16, 603-614

Kristek F., Gerova M., Devat L., Varga I. (1996): Cardiac hypertrophy and vascular remodelling in NO-deficient hypertension. In: The Biology of Nitric Oxide. (Eds. S. Moncada, J. Stamler, S. Gross and E. A. Higgs), pp. 266, Portland Press, London

Kristek F., Gerova M. (2004): Hypotrophy of conduit artery walls of the offspring of nitric oxidedefective rats. Braz. J. Med. Biol. Res. 37, 601-606 http://dx.doi.org/10.1590/S0100-879X2004000400018

Kunes J., Pang S. C., Cantin M., Genest J., Hamet P. (1987): Cardiac and renal hyperplasia in newborn spontaneously hypertensive rats. Clin. Sci. 72, 271-275

Lais L. T., Rios L. L., Boutelle S., DiBona G. F., Brody M. .F. (1977): Arterial pressure development in neonatal and young spontaneously hypertensive rat. Blood Vessels 14, 277-284

Lee R. M. (1985): Vascular changes at the prehypertensive phase in the mesenteric arteries from spontaneously hypertensive rats. Blood Vessels 22, 105-126

Marque V., Kieffer P., Atkinson J., Lartaud-Idjouadiene I. (1999): Elastic properties and composition of the aortic wall in old spontaneously hypertensive rats. Hypertension 34, 415-422

Michel J. B., Heudes D., Michel O., Poitevin P., Philippe M., Scalbert E., Corman B., Levy B.I. (1994): Effect of chronic ANG I-converting enzyme inhibition on aging processes. II. Large arteries. Am. J. Physiol. 267, R124-135

Mulvany M. J., Halpern W. (1977): Contractile properties of small arterial resistance vessels in spontaneously hypertensive and normotensive rats. Circ. Res. 41, 19-26

Okamoto K., Aoki K. (1963): Development of a strain of spontaneously hypertensive rats. Jap. Circ. J. 27, 282-293

http://dx.doi.org/10.1253/jcj.27.282

Olivetti G., Melissari M., Marchetti G., Anversa P. (1982): Quantitative structural changes of the rat thoracic aorta in early spontaneous hypertension. Circ. Res. 51, 19-26

Owens G. K., Schwartz S. M. (1982): Alterations in vascular smooth muscle mass in the spontaneously hypertensive rat. Circ. Res. 51, 280-289

Rizzoni D., Rodella L., Porteri E., Rezzani R., Guelfi D., Piccoli A., Castellano M., Muiesan M.L., Bianchi R., Rosei E. A. (2000): Time course of apoptosis in small resistance arteries of spontaneously hypertensive rats. J. Hypertens. 18, 885-891 http://dx.doi.org/10.1097/00004872-200018070-00010

Rummery N. M., McKenzie K. U. S., Whitworth J. A., Hill C. E. (2002): Decreased endothelial size and connexin expression in rat caudal arteries during hypertension. J. Hypertens 20, $247-253$ http://dx.doi.org/10.1097/00004872-200202000-00014

Saavedra J. M., Correa F. M., Seltzer A., Pinto J. E., Viglione P., Tsutsumi K. (1992): Enhanced angiotensin converting enzyme binding in arteries from spontaneously hypertensive rats. J. Hypertens. 10, 1353-1359

http://dx.doi.org/10.1097/00004872-199211000-00007

Schwartz S. M., Majesky M. W., Dilley R. J. (1990): Vascular remodeling in hypertension and atherosclerosis. In: Hypertension: Pathophysiology, Diagnosis, and Management. (Eds. J. H. Laragh and B. M. Brenner), pp. 521-539, Raven Press Ltd., New York 
Stassen F. R., Raat N. J., Brouwers-Ceiler D. L., Fazzi G. E., Smits J. F., De Mey J. G. (1997): Angiotensin II induces media hypertrophy and hyperreactivity in mesenteric but not epigastric small arteries of the rat. J. Vasc. Res. 34, 289-297 http://dx.doi.org/10.1159/000159236

Touyz R. M. (2005): Intracellular mechanisms involved in vascular remodelling of resistance arteries in hypertension: role of angiotensin II. Exp. Physiol. 90, 449-455 http://dx.doi.org/10.1113/expphysiol.2005.030080

Van Gorp A. W., Schenau D. S., Hoeks A. P., Boudier H. A., de Mey J. G., Reneman R. S. (2000): In spontaneously hypertensive rats alterations in aortic wall properties precede development of hypertension. Am. J. Physiol. Heart Circ. Physiol. 278, H1241-1247

Weibel E. R., Kisteler G. S., Scherle W. F. (1966): Practical stereological methods for morphometric cytology. J. Cell. Biol. 30, 23-38 http://dx.doi.org/10.1083/jcb.30.1.23

Zicha J., Kunes J. (1999): Ontogenetic aspects of hypertension development: Analysis in the rat. Physiol. Rev. 79, $1227-1282$

Received: March 23, 2011

Final version accepted: May 30, 2011 